\title{
Genes for tensin, villin and desmin are linked on mouse Chromosome 1
}

\author{
S.A. Jankowski, D.L. Gumucio \\ Department of Anatomy and Cell Biology, 5793A Medical Sciences II, University of Michigan, Ann Arbor, Michigan 48109-0616, USA
}

Received: 11 May 1995 / Accepted: 27 June 1995

Tensin is an actin-binding protein that is concentrated in submembranous cycoskeletal focal contacts (Weigt et al. 1992). In addition to its three actin-binding domains (Lo et al. 1994), the 200-kDa tensin protein has been found to contain a Src homology 2 (SH2) motif (Davis et al. 1991). This motif mediates protein-protein contacts and is shared by a variety of signal transduction molecules. In addition, tensin can bind to phosphotyrosine-containing proteins (Davis et al. 1991) and can itself be phosphorylated (Davis et al. 1991; Bockholt et al. 1992), suggesting that tensin may be a link between the cytoskeleton and a signal transduction pathway. Indeed, tensin phosphorylation occurs during cell adhesion to extracellular matrix proteins (Bockholt and Burridge 1993).

The cDNA for chick (Gallus gallus) tensin is the only previously reported clone for tensin (Lo et al. 1994). This 7.1-kb cDNA, isolated from a chick cardiac muscle library, contains an open reading frame (ORF) encoding 1744 amino acids, parts of which demonstrate partial homology with a number of previously isolated cytoskeletal proteins (Lo et al. 1994). We now report the isolation of a 643-bp rat kidney partial cDNA clone with homology to the $3^{\prime}$ end of the chick ORF and a portion of the $3^{\prime}$ untranslated region (UTR). Using this clone, we have mapped both the mouse and the human tensin genes.

Polymerase chain reaction (PCR) amplification of singlestranded rat kidney cDNA with primers designed to amplify the rat fibroblast growth factor receptor 1 (FGFR-1) mRNA resulted in the amplification of an additional band smaller than the expected product. Sequencing of the cloned product revealed that both ends contained homology to the $5^{\prime}$ PCR primer. A search of GENBANK showed that the 643-bp cDNA clone is highly homologous to the chick tensin gene: the first $325 \mathrm{bp}$ of nucleotide sequence are $78 \%$ identical with the $3^{\prime}$ end of the chick tensin ORF. An additional 62-bp motif within the putative UTR is $70 \%$ identical to sequence within the chick tensin $3^{\prime}$ UTR (although the spacing between the end of the ORF and this conserved sequence motif in the UTR is not conserved between rat and chick). The degree of conservation of this untranslated region suggests that it may be functionally important. In addition, the first 35 bp of the rat cDNA is $86 \%$ identical with the last 35 bp of a recently reported 177 -bp human expressed sequence tag (Genbank accession number T11757; Liew et al. 1994); this human clone is $73 \%$ identical over $139 \mathrm{bp}$ to chick tensin. Further analysis of the rat cDNA revealed a potential ORF that begins at the second nucleotide in the clone and extends to the 322 nd. Translation of this ORF would result in a peptide with $87 \%$ identity (93/107 amino acids) to the C-terminal end of the chick tensin protein. Therefore, this clone is most likely a partial cDNA for the rat tensin gene.

When the 643-bp rat cDNA was used as a probe for Southern analysis of mouse genomic DNA, a single band was observed. To

Correspondence to: D. Gumucio map this gene, restriction length polymorphisms (RFLPs) were sought between C57BL/6J and $M$. spretus. An RFLP detected upon SacI digestion (Fig. 1a) was used to screen a mapping panel of genomic DNA from The Jackson Laboratory backcross panel, BSS (backcrossed to $M$. spretus; Rowe et al. 1994). The resulting blots were hybridized at $55^{\circ} \mathrm{C}$ to the cDNA clone. Analysis of the data established the location of the mouse tensin gene (Tns) on Chromosome (Chr) 1 between DIBirl3 and DIBir 14, distal to Crygb but proximal to DIMit8. The distances between genes in this region on the BSS panel are therefore as follows: $C r y g b-(3.19$ $\pm 1.81)-D 1 B i r 13-(1.06 \pm 1.06)-T n s-(4.25 \pm 2.08)-D 1 B i r 14-$ (1.06 \pm 1.06)-D1Mit8 (Fig. 1b).

Interestingly, the mouse tensin gene maps within the same region of Chr 1 as two additional genes encoding cytoskeletal proteins, villin (Vil) and desmin (Des) (Seldin et al. 1993). Villin, also an actin-binding protein, is purported to play a role in the morphogenesis of microvilli in epithelial cells that develop a brush border (Friederich et al. 1990). Desmin is an intermediate filament found in a variety of muscle types and is believed to contribute to linkage of cellular organelles to the cytoskeleton or to link actin to the muscle plasma membrane (Tokuyasu et al. 1985). Although all three proteins may function, in part, by binding actin, and all map to the same region of Chr 1 in the mouse, the genes for these proteins are not structurally related.

Also localized to this region of mouse Chr 1 is a cytogenetically detectable deletion produced by maternal X-irradiation (Epstein et al. 1991). The deletion was recovered in heterozygous progeny that exhibited growth retardation and white spotting and was first characterized as a severe allele of the splotch mutation, splotch-retarded, $S p l^{r}$. Since this deletion [Del(1)Sp $\left.{ }^{r} 3 \mathrm{H}\right]$ (Beechey and Searle 1986) encompasses Pax3, Vil, Des, Inha, and Akp3, we tested whether the tensin gene was also included in the deletion region. Comparative Southern hybridization of genomic DNA from both $S p^{r}$ and C57BL/6J mice with the rat cDNA, a mouse villin probe (L. Dunbar, unpublished), and a mouse CCK probe (L. Samuelson, unpublished) was performed (Fig. 2). Quantitation of the resulting bands demonstrated that villin, but not tensin, is present at a reduced level as compared with $\mathrm{CCK}$ in the $S p^{r}$ genome, while all three are present in equal copy numbers in the C57BL/6J genome. Because the deletion contains $A k p 3$ (Epstein et al. 1991), which lies distal to D1Mit8 (Seldin et al. 1993), tensin most likely lies proximal to the deletion, between Vil and Crygb.

The region of mouse $\mathrm{Chr} 1$ to which the tensin gene maps is homologous with human Chr 2 (2q-31-37; Seldin et al. 1993). To establish that the tensin gene does indeed map to human $\mathrm{Chr} 2$, the rat DNA probe was hybridized to a HindIII-digested panel of genomic DNA from both human/mouse and human/hamster somatic cell hybrids. Five bands, 8.5, 8.3, 5.7, 4.6, and $3.5 \mathrm{~kb}$ in length, were detected in human DNA (data not shown). Of these, two ( 8.3 and $4.6 \mathrm{~kb}$ ) were detected in the hybrid containing human $\mathrm{Chr} 2$, and presumably represent the human homolog of the tensin 
12

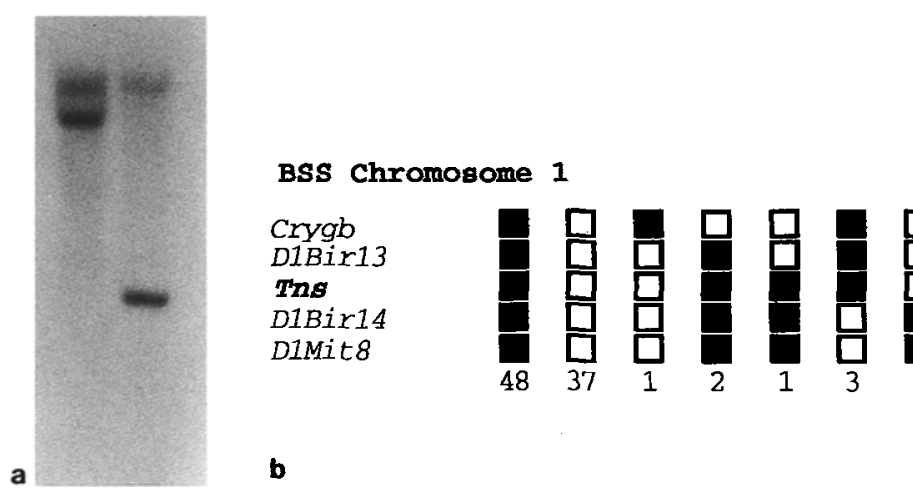

Fig. 1. (a) Hybridization of a Southern blot of $10 \mu \mathrm{g}$ SacI-digested genomic DNA from C57BL/6J (lane 1) and $M$. spretus (lane 2) with the rat tensin cDNA resulted in the detection of a polymorphism that was used for haplotype mapping. (b) The resulting haplotype distribution, which included 94 backcross progeny, is shown with filled boxes representing

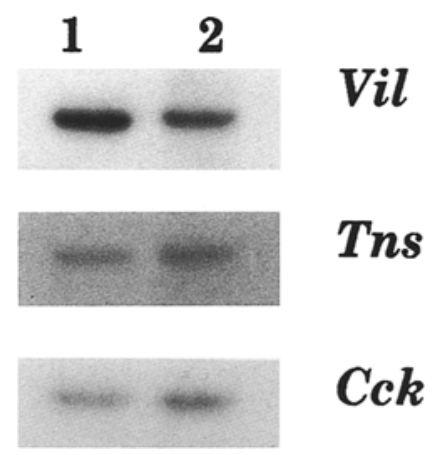

Fig. 2. Genomic DNA from C57BL/6J (lane 1) and $S p^{r}$ (lane 2) was digested with HindIII, blotted, and hybridized sequentially with a partial cDNA probe for the mouse villin and rat tensin genes, and a segment of intron 2 from the mouse cholecystokinin gene. The resulting bands were quantified with a BioRad GS-250 Molecular Imager and Phosphor Analysis/PC image analysis software. Comparison of $\mathrm{C} 57 \mathrm{BL} / 6 \mathrm{~J} / \mathrm{Sp}^{r}$ signal ratios (vil $=1.87$; ten $=0.56$; $\mathrm{cck}=0.59$ ) demonstrates that, unlike villin, tensin does not map within the splotch deletion region.

gene, confirming its predicted location. Of the remaining three bands, the smallest was detected in multiple hybrids, and the others were not detected in any of the hybrids, perhaps because of the weak hybridization signal of these bands compared with the mouse and hamster bands. Localization of the mouse tensin gene to $\mathrm{Chr}$ 1 in a region homologous to human Chr 2 supports localization of the orthologous human tensin (TNS) gene to Chr 2. The detection of additional bands within the human genome may be due to a more limited homology with the region of the tensin gene encompassed by the 643-bp partial cDNA fragment.

Acknowledgments. The authors thank the members of The Jackson Laboratory for the genomic DNA from the BSS backcross panel, Mary Barter and Lucy Rowe for the typing information, advice, and haplotype figure, and Lois Maltais for her help with mouse and human nomenclature. We also thank Dr. Peter Glenister of the MRC Radiobiology Unit, United Kingdom, for providing the splotch-retarded mice. We are grateful to Drs. laura Dunbar and Linda Samuelson for supplying the villin and CCK probes, respectively, Kathy Pinson for providing $S p^{r} \mathrm{DNA}$, and Dr. Miriam Meisler for supplying the filters containing the somatic cell hybrid DNA. S.A. Jankowski is supported by National Research Service Award DK09159. This work has been supported by National Institutes of Health heterozygous loci and open boxes representing loci homozygous for the $M$. spretus allele. The number of animals observed with each haplotype is shown below the column: map distances (in $\mathrm{cM}$ ) were calculated from the recombination fraction observed for the typed progeny.

Grant R29-HD28620 (D.L. Gumucio). The sequence of the 643-bp rat tensin cDNA has been submitted to GENBANK (accession \#U26310). The designation Tns (tensin) has been approved by the International Committees on Mouse and Human Genetic Nomenclature.

\section{References}

Beechey, C.Y., Searle, A.G. (1986) Mutations at the Sp locus. Mouse Newslet. 75, 28.

Bockholt, S.M., Burridge, K. (1993). Cell spreading on extracellular matrix proteins induces tyrosine phosphorylation of tensin. J. Biol. Chem. 268, 14565-14567.

Bockholt, S.M., Otey, C.A., Glenney, J.J., Burridge, K. (1992). Localization of a $215-\mathrm{kDa}$ tyrosine-phosphorylated protein that cross-reacts with tensin antibodies. Exp. Cell Res. 203, 39-46.

Davis, S., Lu, M.L., Lo, S.H., Lin, S., Butler, J.A., Druker, B.J., Roberts, T.M., An, Q., Chen, L.B. (1991). Presence of an SH2 domain in the actin-binding protein tensin. Science 252, 712-715.

Epstein, D.J., Malo, D., Vekemans, M., Gros, P. (1991). Molecular characterization of a deletion encompassing the splotch mutation on mouse chromosome 1. Genomics 10, 89-93.

Friederich, E., Pringault, E., Arpin, M., Louvard, D. (1990). From the structure to the function of villin, an actin-binding protein of the brush border. Bioessays 12, 403-408.

Liew, C.C., Hwang, D.M., Fung, Y.W., Laurenssen, C., Cukerman, E., Tsui, S., Lee, C.Y. (1994). A catalogue of genes in the cardiovascular system as identified by expressed sequence tags. Proc. Natl. Acad. Sci. USA 91, 10645-10649.

Lo, S.H., An, Q., Bao, S., Wong, W.K., Liu, Y., Janmey, P.A., Hartwig, J.H., Chen, L.B. (1994a). Molecular cloning of chick cardiac muscle tensin. Full-length cDNA sequence, expression, and characterization. J. Biol. Chem. 269, 22310-22319.

Lo, S.H., Janmey, P.A., Hartwig, J.H., Chen, L.B. (1994b). Interactions of tensin with actin and identification of its three distinct actin-binding domains. J. Cell Biol. 125, 1067-1075.

Rowe, L.B., Nadeau, J.H., Turner, R., Frankel, W.N., Letts, V.A., Eppig, J.T., Ko, M.S., Thurston, S.J., Birkenmeier, E.H. (1994). Maps from two interspecific backeross DNA panels available as a community genetic mapping resource. Mamm. Genome 5, 253-274.

Seldin, M.F., Hunter, K., Watson, M.L. (1993). Encyclopedia of the mouse genome III. October 1993. Mouse Chromosome 1. Mamm. Genome (Suppl.), S10-S30.

Tokuyasu, K.T., Maher, P.A., Dutton, A.H., Singer, S.J. (1985). Intermediate filaments in skeletal and cardiac muscle tissue in embryonic and adult chicken. Ann. N.Y. Acad. Sci. 455, 200-212.

Weigt, C., Gaertner, A., Wegner, A., Korte, H., Meyer, H.E. (1992). Occurrence of an actin-inserting domain in tensin. J. Mol. Biol. 227, 593595. 